\title{
Thinking about populations and races in time
}

Roberta L. Millstein

Department of Philosophy

University of California, Davis

One Shields Ave

Davis, CA 95616

RLMillstein@UCDavis.edu

\section{Forthcoming in Studies in History and Philosophy of Biological and Biomedical Sciences for a special issue, "Genomics and Philosophy of Race." There may be small changes between this version and the final published version.}

\begin{abstract}
Biologists and philosophers have offered differing concepts of biological race. That is, they have offered different candidates for what a biological correlate of race might be; for example, races might be subspecies, clades, lineages, ecotypes, or genetic clusters. One thing that is striking about each of these proposals is that they all depend on a concept of population. Indeed, some authors have explicitly characterized races in terms of populations. However, including the concept of population into concepts of race raises three puzzles, all having to do with time. In this paper, I extend the causal interactionist population concept (CIPG) by introducing some simple assumptions about how to understand populations through time. These assumptions help to shed light on the three puzzles, and in the process show that if we want to understand races in terms of populations, we will need to revise our concept(s) of race.
\end{abstract}

\section{Introduction}

Are biological races a legitimate biological category in Homo sapiens? To answer this question, 
biologists and philosophers have had to characterize "biological race." ${ }^{1}$ Such characterizations differ, but notably, the concept of population plays a central role in many of them. For example:

- Races as genetically different - "A geneticist can define races as populations that differ from each other in the frequencies of certain genes" (Dobzhansky 1941, 162; emphasis added).

- Races as subspecies - a race is "a geographically localized subdivision of the species, which differs genetically and taxonomically from other subdivisions of the species [and is]... composed of a number of genetically distinct populations (Mayr 1942, 106; emphasis added).

- Races as lineages - a race is "a distinct evolutionary lineage within a species" (Templeton 1998, 632), but "recurrent genetic interchange among Old World human populations" would mean that human races do not exist (Templeton 1998, 636; emphasis added).

- Races as clades - "races are ancestor-descendant sequences of breeding populations, or groups of such sequences, that share a common origin" (Andreasen 1998, 200; emphasis added).

- Races as ecotypes - races are "local populations adapted to particular environments" (Pigliucci and Kaplan 2003, 1161; emphasis added).

\footnotetext{
${ }^{1}$ By specifying biological race concepts, I mean to distinguish biological concepts from "commonsense" or "social" concepts of race. This is not to say that the biological concepts are somehow asocial, but rather that such concepts have specifically sought to identify a biological correlate for race.
} 
- Races as populations - 'race,' in its current U.S. meaning, is a proper name for a particular set of human populations (Spencer 2014a).

- Races as population groups - a race is a group of populations that exhibits a distinctive pattern of genetically transmitted phenotypic characters that corresponds to the group's geographical ancestry and belongs to a biological line of descent initiated by a geographically separated and reproductively isolated founding population (Hardimon manuscript).

In this paper, I will neither take a stand on which biological race concept, if any, is viable, nor discuss the question of whether, given a defensible concept (or concepts) of race, races exist in Homo sapiens. Rather, I examine three puzzles that result from including a population concept in a race concept:

1. Population membership at a time: Can a person be a member of more than one population at a time, as we would say that a person can be a member of more than one race?

2. Forward-looking vs. backward-looking concepts: Is "race" a "backward-looking" concept (i.e., looking to the past), and is "population" a "forward-looking" concept (i.e., looking to the future)? If so, have confusions been introduced by characterizing "race" in terms of "population"?

3. Status of genetic clusters: Can genetic clusters (see, e.g., Rosenberg et al. 2002, 2005), the result of interactions from different points in the past, be understood as delineations of 
populations or races or both?

Note that all three puzzles involve time. Among race scholars, Lisa Gannett has usefully drawn attention to the importance of time in thinking about races and populations; I thus use her work as a springboard. She argues that thinking about time supports the view that there are multiple legitimate concepts of "population," depending on research context (Gannett 2003). For example, one researcher might wish to predict the future composition of a particular population's gene pool, given the population's current size, genetic composition, breeding structure, and rates of mutation and migration. Such a researcher, Gannett suggests, would use a forward-looking population concept, relying on breeding units to delineate the population; the organisms that are interbreeding will determine the future gene pool, regardless of their past origin(s). Another researcher might want to compare adaptive differences among the same organisms - organisms, let us suppose, that have origins in different environments. Such a researcher, Gannett suggests, would use a backward-looking population concept, relying on ancestral relations to delineate the population; the researcher would not want to lump together in the same population organisms that had undergone different selection processes, even if they were now interbreeding. Thus, on Gannett's account, there are at least two different population concepts that a researcher might use, depending on the research question she was seeking to answer, one forward looking (using interbreeding as a criterion) and one backward looking (using ancestral relations as a criterion). ${ }^{2}$

In a paper on the use of race in pharmacogenetics research, Gannett further states:

\footnotetext{
2 Baum (1998) makes similar claims with respect to species concepts, distinguishing between a forward-looking interbreeding species concept and a backward-looking genealogical species concept.
} 
Genetic clustering tells us something about the ways in which individual genomes have been shaped by past, even long past, reproductive choices and environments. But what is it to say, further, that these genetic clusters themselves represent biological groups whose component individuals share "genetic ancestry" or a common "ancestral geographical origin"? Which ancestors (matrilineal, patrilineal, recent, remote)? Which geographical locations (country, city, village, riverbank, valley, continent, island)? Research context determines the geographical scale or historical period of interest (2005, 1237-8; emphasis added).

Here, Gannett seems to be suggesting that there is no privileged point in time (and no privileged place) that would univocally determine a person's "race."

Contrary to Gannett's pluralism about populations, Millstein (2009, 2010a) defends a monist population concept: the causal interactionist population concept (CIPC). ${ }^{3}$ In what follows, I extend (and slightly modify) the CIPC in order to show more explicitly how it incorporates time, using some relatively simple assumptions about how to understand populations through time. In light of this elaboration, I agree with Gannett that there is no privileged point in time that would univocally constitute a person's "race," but disagree with her claim that looking-forward and looking-backward require different concepts of population. I then seek to shed light on the three puzzles I outlined above.

\footnotetext{
${ }^{3}$ However, it is a defeasible monism (Millstein 2010b). Note that I will simply presume the CIPC here; see Millstein (2009, 2010ab, 2014) for a defense. I recognize that the CIPC does not capture all biologists' utterances of the term "population" in ecological and evolutionary contexts, but it avoids difficulties encountered by other population concepts, e.g., difficulties with describing and predicting evolutionary dynamics. See Barker and Velasco (2013) and Stegenga (2014) for more recent defenses of population pluralism, discussion of which would take me afield from the arguments of this paper.
} 
A note before proceeding: The authors cited above do not generally specify what they mean by “population." Or they default to population genetics' definitions of the term, which are themselves not clear and whose ambiguity raises various problems (Millstein 2009). ${ }^{4}$ This ambiguity necessitates the use of an articulated and defended conception of "population"; thus, I use the concept that I have previously defended, the CIPC. I suspect that it would possible to make similar points with an alternate concept of population (e.g., the population-as-deme concept discussed in Millstein 2014), but I have not fully explored that possibility, and I do not explore it here.

\section{Incorporating Time into the Gausal Interactionist Population Concept (GIPG)}

I begin by outlining the basic elements of the CIPC (see Millstein 2010a for further details). According to the CIPC, in ecological and evolutionary contexts ${ }^{5}$ populations consist of at least two conspecific organisms who, over the course of a generation, are actually engaged in survival or reproductive interactions, or both. Reproductive interactions include both unsuccessful and successful matings (interbreeding), as well as offspring rearing. Survival interactions are almost as broad as Darwin's “struggle for existence," including competition for limited resources as well as cooperation. Social interactions are not a separate category, but may fall into either or both of the other two categories; social interactions that do not affect survival or reproduction are not relevant evolutionarily or ecologically.

\footnotetext{
${ }^{4}$ See also Kaplan et al. (THIS ISSUE) for discussion of a race debate where problems are caused by a failure to offer a defensible way of recognizing biologically meaningful populations.

5 That is, the CIPG makes no claims about the concept of population in other domains, where it will likely differ at least in some if not many respects. My implicit claim here is that the proposed biological notions of race arise in ecological and evolutionary contexts, a claim that I take to be uncontroversial.
} 
The CIPG further specifies that the boundaries of the population are the largest grouping where the rates of interaction ${ }^{6}$ are much higher within the grouping than outside. Thus, organisms located in the same spatial area (including recent migrants) are part of the population if and only if they are interacting with other conspecifics. If a later grouping is causally connected by survival and/or reproductive interactions to an earlier grouping, then it is the same population, although fusing, splitting, and budding are in this essay newly introduced as exceptions to this general rule (see discussion below). Thus, populations are continuous through time even as organisms die and new ones are born, just as organisms themselves are continuous through time even as cells die and new ones are born.

To understand populations through time, we need to know how populations begin (are "born"), how populations end ("die"), how to handle population splitting and "budding," and how to handle populations fusing. The CIPC specifies that new populations generally begin with interacting founders who are no longer interacting with others in the population, as might occur with geographical separation, and that populations end with the death of the last organism in the population, if not sooner (Millstein 2009). ${ }^{7}$ But more needs to be said. What follows are some simple and straightforward proposals for thinking about populations through time. These are intended to be uncontroversial and indeed unsurprising given longstanding biological practice. ${ }^{8}$ What I hope to be more surprising are the consequences of accepting these proposals, as described in section 3).

\footnotetext{
${ }^{6} \mathrm{Jim}$ Griesemer has suggested to me that the strengths of interactions, and not just their rates, might be important for the constitution of a population (consider, for example, a rare reproductive event that nonetheless contributes new and important alleles to the population). This is a topic worthy of further consideration; I find the suggestion appealing, although I worry about how one would measure relative strengths.

${ }^{7}$ Lack of interaction between organisms might also be the end of a population; other ways are shown below. Previous incarnations of the CIPC are not committed to any particular way that individual populations cease to exist, only that they, like individual organisms, eventually do cease to exist.

${ }^{8}$ See also Sober (2000, 157-159), who makes similar points.
} 
2.1. Populations fusing. If the organisms in one population $a$ begin interacting with the organisms in a second population $b$, they will form a new population $c{ }^{9}$ (See Figure 1). This follows straightforwardly from the core interactionist aspect of the CIPC, which relies on survival and reproductive interactions to delineate populations. Populations $a$ and $b$ cease to exist when their constituent organisms begin interacting, and a new population $c$, which could not reasonably be identified with either $a$ or $b$ and having a new dynamics and trajectory, is formed from the interactions of the former members of $a$ and $b$.

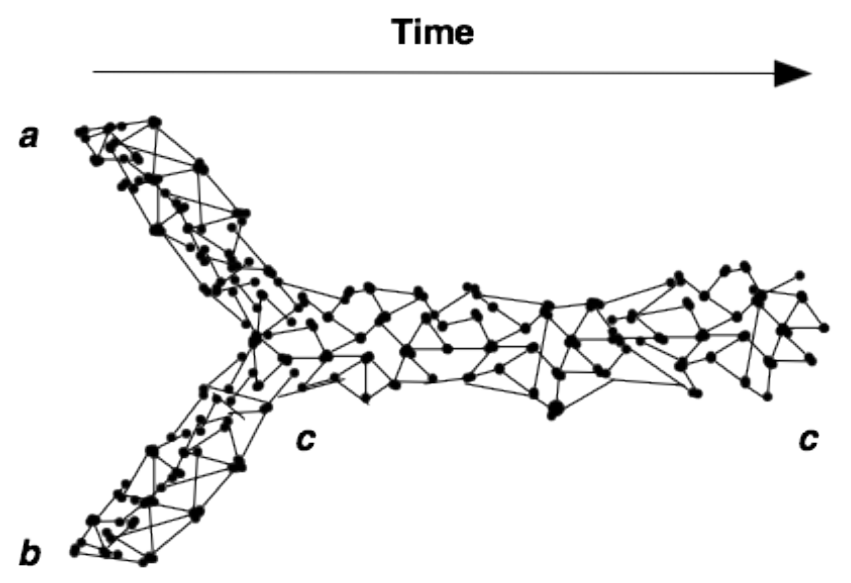

Figure 1: Populations fusing. Populations $\mathrm{a}$ and $\mathrm{b}$ fuse to form a new population $\mathrm{c}$.

\subsection{Population splitting and budding. Again focusing on interactions, if a significant}

fraction of the organisms in one population $a$ cease interacting with a significant fraction of the rest of the organisms in population $a$, they will form two new populations, $b$ and $c$. This is a splitting of population $a$; population $a$ ceases to exist. (See Figure 2i). If, on the other hand, only a

\footnotetext{
${ }^{9}$ Note fusing necessitates a qualification of the "continuity" aspect of the CIPC from what Millstein (2009, 2010a) previously characterized; that is, this is a case where even though later groupings are causally connected by survival or reproductive interactions to earlier groupings, they are not the same population.
} 
small fraction of the members of $a$ cease interacting with the other members of $a$, we say that a new population $b$ has budded off from population $a$, with population $a$ persisting after the budding. (See Figure 2ii). ${ }^{10}$ In both splitting and budding, one population becomes two. In the budding case, the original population persists, but it does not in the splitting case. Obviously, there is a continuum between splitting and budding. The term "budding" can be reserved for the cases where a truly small fraction has separated off, leaving intermediate, uncertain cases to be considered a "splitting." The reason is that any sort of substantial separation would mean that the new groupings would have a substantially different character (dynamics and trajectory) from what existed before, meaning that the original population has ceased to exist. Still, the populations are related genealogically; for either splitting or budding, we would say that the new populations are descended from the original populations.

\footnotetext{
10 Note that splitting and budding necessitate a qualification of the "continuity" aspect of the CIPC from what Millstein (2009, 2010a) previously characterized; that is, these are cases where even though a later grouping is causally connected by survival or reproductive interactions to an earlier grouping, it may not be the same population.
} 

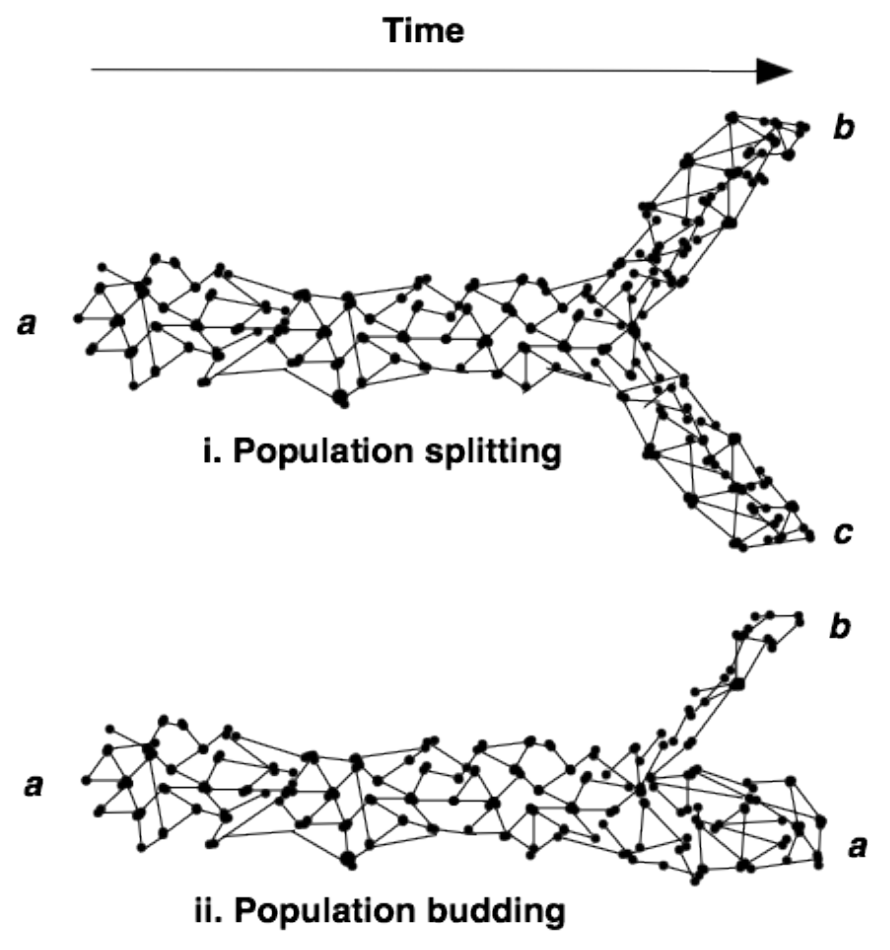

Figure 2: Population splitting and population budding.

i. Population a splits to form populations $b$ and $c$.

ii. Population b buds off from population a; population a persists.

2.3 Limited migration. When an individual organism ceases interacting with the members of one population $a$ and begins interacting with the members of a different population $b$, it ceases being a member of $a$ and becomes a member of population $b$. (See Figure 3). We would still say that the individual was formerly a member of $a$; the individual may have also been descended from $a$. This has been part of the CIPG all along, and is a standard way of handling migration in ecology and evolution. I emphasize it here in order to highlight that an organism's membership in any given population is always relativized to time. That is, we should say that an organism was a member of population $a$ during time span $x$ and a member of population $b$ during time $\operatorname{span} y$. 


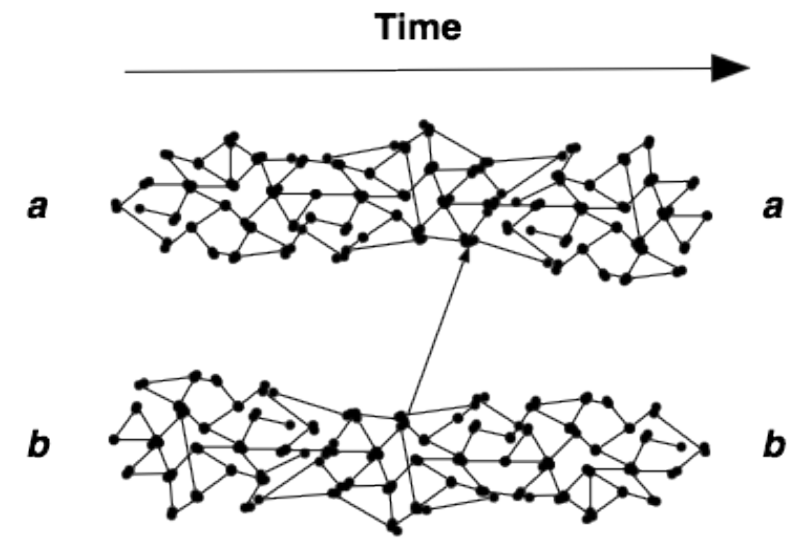

Figure 3: Limited migration. An individual organism leaves population $b$ and becomes a member of population $a$.

2.4 Multiple migrations. Consider the two populations depicted in Figure 3. Suppose that instead of rare migration between the populations, there were frequent migrations between the groupings. In that case, there would be one population, albeit one patchy population (Millstein 2010a), rather than two. (See Figure 4). Because of the interactions between the patches, the fates of the two patches are linked. For example, an advantageous allele arising in one patch may spread into the other patch and become common in the population as a whole. Of course, there is linkage between the populations represented in Figure 3 as well, but to a much lesser degree; the two populations linked by infrequent migration/interaction are a metapopulation (Millstein 2010a). As with the distinction between splitting and budding, there is no sharp dividing line between metapopulations and patchy populations. 


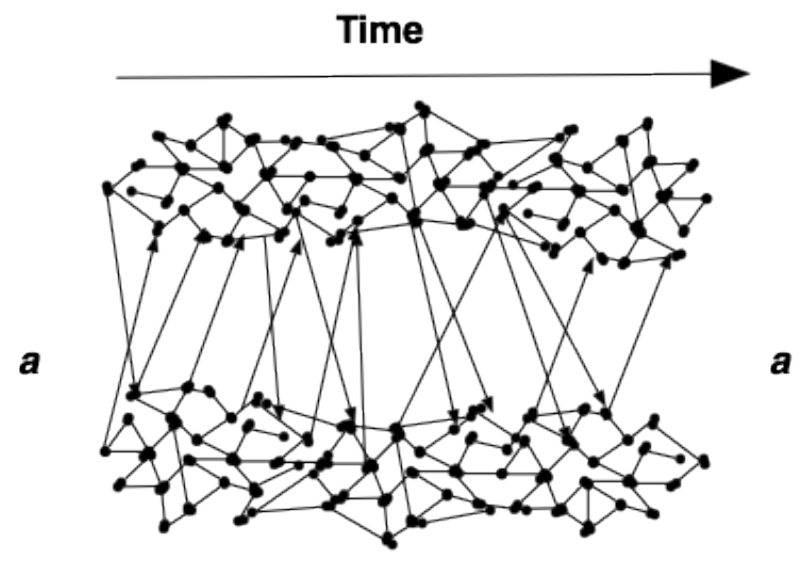

Figure 4: Multiple migrations. What might otherwise be two populations is actually one population, a, because of the interaction of organisms between the patches.

\section{Shedding Light on the Three Puzzles}

These simple, straightforward assumptions help answer our three puzzles, as follows.

3.1. Population membership at a time. Can a person be a member of more than one population, as we would say that a person can be a member of more than one race? In short, no. As discussed in Section 2.3, a person is a member of only one population at a time - the one they are currently interacting with. When they stop interacting with the members of one population and begin interacting with the members of another, they leave the former and join the latter. Thus, a person can be formerly a member of one population and currently a member of a new population. The exception is when many such people are interacting between what would otherwise be separate populations. As discussed in Section 2.4, this is in fact one patchy population, so the person in question is still a member of just one population at a time. 
More precisely, if a person begins interacting with a new population, then that person was a member of population $a$ during time span $x$ and is a member of population $b$ during time span $y$. An offspring of an individual who migrated from $a$ to $b$, is descended from population $a$ but is not a member of population $a$. Similarly, in cases of splitting or budding, offspring of members of the new population are descended from the original population $a$ but are not members of population $a$. In these ways, population membership is relativized to time. ${ }^{11}$

My discussion here might prompt some to think about various possible degenerate cases. For example, suppose a person is going back and forth between populations, e.g., leaving a population to bring food for sale to another population and then returning back to the original population, further supposing that they are the only person, or one of a small number of people, doing so. (Since again, if many such people were engaging in such interactions, there would be one patchy population, not two separate populations). Assuming that the majority of the person's interactions are with the original population, the person's fate is more tightly linked with the original population, thus making that the population to which they belong. On the other hand, suppose the person alternately interacted with the two populations for six months each, so that one might think that there was no reason to say that the person was more a member of one population than the other. Although it might seem counter-intuitive, relativizing to time seems to be the answer here; the person is a member of the first population for a time, then a member of the second population for a time (and not a member of the first), and then upon re-interacting with a first population again, becomes a member of that population for the subsequent time period. Finally, we might consider the case of someone who is interacting with more than one purported population at the same time. It is difficult to come up with examples that would not

${ }^{11}$ Note that this is different from delimiting a population by a certain time period. 
also imply other interactions between the two groupings, making them one patchy population rather than two: the dropping of a nuclear bomb, the creation of a dump site, or the building of a noisy airport. Perhaps there are such cases, but I suspect they are rare. Note that the CIPG is not meant to be a definition spelled out in terms of necessary and sufficient conditions, with counter-examples serving as defeaters. Rather, it is a concept that can be applied to various cases, i.e., various groupings of organisms in nature, ${ }^{12}$ fitting them more or less well. Biology is messy and no concept can or should correct for that.

If we want to maintain the claim that a person can be of more than one race while incorporating a population concept into the race concept, we should likewise be clear about which population they are currently a member of and which populations they are descended from - and when. To do otherwise is to potentially confuse past population membership with present. And we would need to reconcile the fact that someone can change the population that they are a member of (i.e., are a part of), but we generally don't think that someone can change their race.

\subsection{Forward-looking vs. backward-looking concepts. Is 'race' a 'backward-looking' concept?'}

Maybe; I have not taken a stand on that here. But since biological concepts of "race" are often closely tied to "ancestry," at least some of them are. Is "population" a "forward-looking" concept? Not solely, as we have seen. Populations, I argued, can either be backward or forward looking. Contra Gannett, we do not need two different concepts to capture this. Instead, we just need to indicate the relevant time period - present-day or some time in the past.

\footnotetext{
12 Thus the CIPG is in the spirit of Winther et al.'s (THIS ISSUE) "natural population" kind, although there is no reason in principle that one could not try to apply the CIPC to a laboratory population, which would mean that the GIPC could fit two of the three kinds in Winther et al.'s tripartite distinction.
} 
Have confusions been introduced by characterizing "race" in terms of "population"? If race is solely backward looking, then yes, perhaps there are some confusions introduced by thinking about race in terms of populations. For example, a person might be descended from a population that she is no longer interacting with, and thus her fate is no longer intertwined with members of that population in the same way. ${ }^{13}$ Alternatively, we might revise our concept of race to be both forward and backward looking, just as the concept of population is. Perhaps this would not be problematic; perhaps, for example, a researcher might coherently wish to predict what a particular race's gene pool will look like in the future given the population's current size, genetic composition, breeding structure, and rates of mutation and migration. ${ }^{14}$ Such understandings would, however, require some modifications to our current concepts of race. Attending to time makes us realize the necessity for making such modifications.

3.3 Status of genetic clusters. Can genetic clusters, the result of interactions from different points in the past, be understood as delineations of populations or races or both? First, a bit of background: to formulate genetic clusters, a computer program such as STRUCTURE "loosely speaking, identifies subgroups that have distinctive allele frequencies" (Rosenberg et al 2002: 2382). Individuals can be assigned to more than one cluster to varying degrees. Rosenberg et al. found that "individuals from the same predefined population nearly always shared membership coefficients in inferred clusters" (2002, 2382). ${ }^{15}$ In saying that "predefined [population] labels were highly informative

\footnotetext{
13 This example assumes, as do other parts of this paper, that humans are currently divided into multiple populations, an assumption that has been challenged by M.A. Hunter (2014). Relatedly, I also do not wish to deny that in today's interconnected societies that the fates of individuals descended from the same population are totally unlinked; they might, for example, be linked through societal biases.

14 Thanks to Michael Hardimon for this suggestion.

${ }^{15}$ A more recent study reaches similar conclusions: "Using the MS5435 subset, we sought to verify the accuracy of the individual [population] labels and the genotypic alignment of the source data sets. MDS plots of allele-sharingdistances illustrate that in the first two dimensions, indigenous individuals from the same geographic region largely cluster together, and admixed individuals lie between the clusters of their ancestral populations. These results
} 
about membership in genetic clusters," Rosenberg et al. seem to be suggesting that similar membership across genetic clusters correspond reasonably well to populations. They also found that when the computer program was instructed to find five clusters, those five clusters "corresponded largely to major geographic regions" (2002, 2382); roughly, these were continental regions, divided by "major physical barriers" such as oceans, the Himalayas, and the Sahara (2002, 2384). They avoid referring to the larger continental groups as "populations" or "races," but others, notably Wade (2002), do not hesitate to identify those five continental groups with races. ${ }^{16}$

To return to the question asked at the outset of this subsection, two possible interpretations of Rosenberg et al.'s (2002) results are that (1) the researchers have found evidence that most of the individuals from a given "predefined population"17 are a sample of a genuine biological population (rather than, e.g., a sample of multiple biological populations), since individuals from the "predefined populations" have similar percentage membership in genetic clusters, and/or (2) the researchers have found evidence for the reality of human races (that, contra many scholars, human races really exist) by finding genetic clusters that roughly correspond to the continental origin of study subjects. ${ }^{18}$ Interpretation (1) is a claim about human populations at a local (subcontinental) level, whereas interpretation (2) is a claim about human races at the (roughly) continental level.

support the inference that the individual [population] labels in the aligned human data are correct" (Pemberton et al. 2013, 900).

${ }^{16}$ Feldman (2010) argues for the use of the term "ancestry group."

${ }^{17}$ It is somewhat unclear how these were defined, but it seems that some combination of language, geography, and self-identification often played a role (Noah Rosenberg, personal communication).

18 Note that I am not endorsing these interpretations myself; Rosenberg et al. do not seem to be endorsing (2), as Wade does, but they may be endorsing (1). 
If (1) is correct, then the larger continental groupings, which consist of numerous such populations, would not be populations themselves, at least according to the CIPG. ${ }^{19}$ The CIPG holds that the boundaries of a population are the largest grouping where the rates of interaction are much higher within the grouping than outside. Thus, if the interactions were such that populations manifested at a more local scale, then the interactions would not be sufficient to form populations at the continental level, ${ }^{20}$ although perhaps there might be sufficient interactions between the populations for the continental groupings to form metapopulations (or metametapopulations). Therefore, if (1) is true, then the genetic clusters do not correspond to populations (but perhaps similar percentage membership in clusters does), and there is a problem for the view that races (if they are indicated by genetic clusters, as (2) suggests) are in some sense populations.

However, there is a larger problem here, suggested by the quotation from Gannett above. Genetic clusters contain alleles that are the result of interactions from different points in time. Thus, it is ambiguous to suggest that similar percentage membership in clusters is evidence for human populations (interpretation 1), or that the genetic clusters are evidence for human races (interpretation 2), because human genetic clusters probably include genetic characteristics that originate from interactions within multiple populations from different points in the past: populations that have split, budded, fused, or been affected by immigration. The mere fact that the "predefined" populations often correspond to multiple genetic clusters ("continents") itself

\footnotetext{
${ }^{19}$ Spencer (2014b) defends a concept of population on which these larger groupings would count as populations.

${ }^{20}$ In other words, the CIPG would suggest that there are either populations at the continental level or at more local groupings, but not both. If there are enough interactions at the continental level to form populations at that level, then there are no populations at a more local level (but perhaps there are patches).
} 
suggests that. ${ }^{21}$ In other words, are genetic clusters supposed to be evidence for current populations/races or past ones, and if past, at which points in the past? The clusters themselves do not provide this information, and so such claims cannot be inferred merely from considering cluster membership. ${ }^{2223}$ Thus, these sorts of time-ambiguous claims about populations and races, represented by interpretations (1) and (2), should be avoided altogether.

To say that genetic clusters are evidence for "races" (interpretation 2) is to pick out a time period when differentiation between peoples of different continents occurred. However, it is questionable whether there ever was such a time period; there is reason to think that human population structure is not that neat and tidy (Hunley, Healey, and Long 2009). We have good reason to doubt any view that explicitly suggests or implicitly implies that human history is characterized by isolated populations unaffected by migration, budding/splitting, and fusing. Invoking notions of population in race concepts without explicit mention of relevant time period risks implying that the populations in question are easily identified, persistent, and relatively static entities through long stretches of time. ${ }^{24}$

Even if there were a time period when differentiation between peoples of different continents occurred, there seems to be no principled reason why it would be any more significant evolutionarily or phenotypically than any other time period. For example, Moreno-Estrada et al.

\footnotetext{
${ }^{21}$ In Templeton's words: “...it is not surprising that when Behar et al. (2010) sampled Old World populations more finely and used the computer program STRUCTURE, most individuals showed significant genetic inputs from two or more populations, indicating that most human individuals have mixed ancestries and do not belong to a 'pure' group" (2013, 268).

${ }^{22}$ See Winther et al. (THIS ISSUE) for a discussion of similar problems that can be encountered when trying to infer populations from genetic clusters.

${ }_{23}$ On the other hand, methods used by biologists such as Ralph and Coop (2013) are able to infer something about the time and place of common origin, by looking at the lengths of shared genomic segments (which get shorter as you go further back in time, due to recombination).

24 See Winther and Kaplan (2013) for a discussion of how migration, splitting, and fusion affect tree topology.
} 
(2014) show that in Mexico, some populations are as differentiated as "Europeans" are from

"East Asians," with functional and medically relevant alleles that are rare or absent elsewhere.

Of course, researchers might be interested in a particular evolutionary change (genotypic or

phenotypic), and their interest in that particular change might dictate that they examine a

particular period of time, but that is just to prove Gannett's (2005) point that there are historical

periods of interest that depend on research context, but no historical period that is categorically

important regardless of research context.

Likewise, to say that similar percentage membership in genetic clusters is evidence for populations (interpretation 1) is to pick out a certain time period as significant; but again, why would one point in time be more significant than any other? Finally, since we are all likely to be descended from multiple populations, it is ambiguous to speak of certain people or populations as "mixed" or "admixed" (e.g., the aforementioned study by Moreno-Estrada et al. differentiates between "admixed" populations and indigenous ones). To do so is to make a claim about a person being descended from more than one population - but which populations? ${ }^{25}$ Without specifying the time period, we give unwarranted significance to certain populations we are descended from, and too often those end up being continental groups from unspecified time periods. ${ }^{26}$

\section{Conclusion}

\footnotetext{
${ }_{25}$ Along similar lines, Lorusso and Bacchini note, “...when you want to search for percentages of genetic ancestry in a particular individual, you need to compare her DNA variation with that of samples coming from populations presumed to be proxies for her ancestral populations. However, the choice of the proxy populations is at least partially arbitrary, therefore each attribution of a specific genetic ancestry to an individual is in itself ambiguous" (THIS ISSUE, PG; emphasis in original, references deleted).

${ }^{26}$ See Verdu and Rosenberg (2011) and Liang and Nielsen (2014) for methods that seek to take into account the history of population admixtures.
} 
In short, we need to pay attention to time. Doing so shows that it may be challenging to incorporate a population concept into a race concept.

I have shown this by making some simple and straightforward assumptions about how to think about populations through time. These assumptions help to shed light on the three puzzles described at the outset of this paper. First, whereas on most accounts of race, a person can be a member of more than one race, a person can be a member of only one population at a time, although they may be descended from more than one population or change their population during their lifetime. Population membership is always relativized to time. Race concepts that incorporate a population concept should do likewise. Second, we do not need different concepts to handle forward-looking and backward-looking populations; again, we just need to specify the time period under consideration for the population in question. Race concepts that incorporate a population concept may likewise need to encompass different points in history as well as future times. Third, we need to be very careful about inferring races and populations from genetic clusters, recognizing that the data reflect population structure from many points in time and resisting the temptation to privilege some points in time over others.

In other words, if we want to understand races in terms of populations, our race concepts may be different than we thought they were, in particular, containing explicit attention to matters of time. Conceiving of races as populations without proper attention to time yields problematic results. Although it may seem as though I have argued for a stronger claim (e.g., that races are not populations), I offer my arguments here in the same spirit that Spencer (THIS ISSUE) does, namely, in the hope of moving the debate forward. If I am right, we either need a better concept 
of race or a better concept of population, or both. Of course, having defended the CIPC in several papers (Millstein 2009, 2010ab, 2014), my own view is that it is our concepts of race that are in need of revision.

\section{Acknowledgements}

Thanks to the Griesemer/Millstein Lab for helpful feedback on the presentation that preceded this paper, and to my philosophy of biology graduate seminar in Fall 2013 for working through many papers in the philosophy of race from a biological point of view with me. Thanks also to members of the IHR Genomics and Philosophy of Race Research Cluster for helpful discussion throughout 2013-2014 - particularly to Rasmus Grønfeldt Winther for his tireless organizing and active engagement with each presentation, including my own - and to Michael Hardimon, Quayshawn Spencer, and three anonymous referees for helpful comments on the manuscript. 


\section{References}

Andreasen, Robin O. 1998. A new perspective on the race debate. The British Fournal for the Philosophy of Science 49 (2):199-225.

Barker, Matthew J., and Joel D. Velasco. 2013. Deep Conventionalism about Evolutionary Groups. Philosophy of Science 80:971-982.

Baum, David A. 1998. Individuality and the existence of species through time. Systematic Biology 47 (4):641-653.

Dobzhansky, Theodosius. 1941. The race concept in biology. The Scientific Monthly 52:161-165.

Feldman, Marcus W. 2010. The Biology of Ancestry: DNA, Genomic Variation, and Race. In Doing Race: 21 Essays for the 21st Century, edited by H. R. Markus and P. M. L. Moya. New York: W. W. Norton.

Gannett, Lisa. 2003. Making populations: Bounding genes in space and in time. Philosophy of Science 70 (5):989-1001.

---. 2005. Group categories in pharmacogenetics research. Philosophy of Science 72 (5):1232-1247.

Hardimon, Michael. manuscript. Rethinking Race: The Case for Deflationary Realism.

Hunley, Keith L, Meghan E Healy, and Jeffrey C Long. 2009. The global pattern of gene identity variation reveals a history of long-range migrations, bottlenecks, and local mate exchange: implications for biological race. American Fournal of Physical Anthropology 139 (1):35-46.

Hunter, M. A. 2014. Populations, Individuals and Biological Race. Presented at the Biennial Meeting of the Philosophy of Science Association. Chicago, IL. 
Kaplan, Jonathan, Massimo Pigliucci, and Josh Banta. THIS ISSUE. Gould on Morton, Redux: What can the debate reveal about the limits of data? Studies in History and Philosophy of Biological and Biomedical Sciences TBD:TBD-TBD.

Liang, Mason, and Rasmus Nielsen. 2014. Understanding Admixture Fractions. bioRxiv:008078.

Lorusso, Ludovica, and Fabio Bacchini. THIS ISSUE. A Reconsideration of the Role of Self-Identified Races in Epidemiology and Biomedical Research. Studies in History and Philosophy of Biological and Biomedical Sciences TBD:TBD-TBD.

Mayr, Ernst. 1942. Systematics and the origin of species, from the viewpoint of a zoologist: Harvard University Press.

---. 1996. What Is a Species, and What Is Not? Philosophy of Science 63 (2):262-277.

Millstein, Roberta L. 2009. Populations as Individuals. Biological Theory: Integrating Development, Evolution and Cognition 4 (3):267-273.

---. 2010a. The Concepts of Population and Metapopulation in Evolutionary Biology and Ecology. In Evolution Since Darwin: The First 150 Years, edited by M. A. Bell, D. J. Futuyma, W. F. Eanes and J. S. Levinton. Sunderland, MA: Sinauer.

---. 2010b. Should We Be Population Pluralists? A Reply to Stegenga. Biological Theory: Integrating Development, Evolution and Cognition 5 (3):271-276.

---. 2014. How the Concept of Population Resolves Concepts of Environment. Philosophy of Science 81:741-755.

Moreno-Estrada, Andrés, Christopher R. Gignoux, Fernández-López, Juan Carlos, Fouad Zakharia, Martin Sikora, Alejandra V. Contreras, Victor Acuña-Alonzo, Karla Sandoval, Celeste Eng, Sandra Romero-Hidalgo, Patricia Ortiz-Tello, Victoria Robles, Eimear E. Kenny, Ismael NuñoArana, Rodrigo Barquera-Lozano, Gastón Macín-Pérez, Julio Granados-Arriola, Scott Huntsman, Joshua M. Galanter, Marc Via, Jean G. Ford, Rocío Chapela, William Rodriguez- 
Cintron, Jose R. Rodríguez-Santana, Isabelle Romieu, Juan José Sienra-Monge, Blanca del Rio Navarro, Stephanie J. London, Andrés Ruiz-Linares, Rodrigo Garcia-Herrera, Karol Estrada, Alfredo Hidalgo-Miranda, Gerardo Jimenez-Sanchez, Alessandra Carnevale, Xavier Soberón, Samuel Canizales-Quinteros, Héctor Rangel-Villalobos, Irma Silva-Zolezzi, Esteban Gonzalez Burchard, and Carlos D. Bustamante. 2014. The genetics of Mexico recapitulates Native American substructure and affects biomedical traits. Science 344 (6189):1280-1285.

Pemberton, Trevor J, Michael DeGiorgio, and Noah A Rosenberg. 2013. Population Structure in a Comprehensive Genomic Data Set on Human Microsatellite Variation. Genes $\mid$ Genomes | Genetics 3:891-907.

Pigliucci, Massimo, and Jonathan Kaplan. 2003. On the concept of biological race and its applicability to humans. Philosophy of Science 70 (5):1161-1172.

Ralph, Peter, and Graham Coop. 2013. The geography of recent genetic ancestry across Europe. PLoS biology 11 (5):e1001555.

Rosenberg, Noah A, Saurabh Mahajan, Sohini Ramachandran, Chengfeng Zhao, Jonathan K Pritchard, and Marcus W Feldman. 2005. Clines, clusters, and the effect of study design on the inference of human population structure. PLoS genetics 1 (6):e70.

Rosenberg, Noah A, Jonathan K Pritchard, James L Weber, Howard M Cann, Kenneth K Kidd, Lev A Zhivotovsky, and Marcus W Feldman. 2002. Genetic structure of human populations. Science 298 (5602):2381-2385.

Sober, Elliott. 2000. Philosophy of Biology, Second Edition. Boulder, CO: Westview.

Spencer, Quayshawn. 2014a. A Radical Solution to the Race Problem. Philosophy of Science 81:1025-1038.

---. 2014b. Do Humans have Continental Populations? Presented at the Biennial Meeting of the Philosophy of Science Association. Chicago, IL. 
---. THIS ISSUE. Philosophy of Race Meets Population Genetics. Studies in History and Philosophy of Biological and Biomedical Sciences TBD:TBD-TBD.

Stegenga, Jacob. 2014. Population Pluralism and Natural Selection. The British fournal for the Philosophy of Science TBD:TBD-TBD.

Templeton, Alan R. 1998. Human races: a genetic and evolutionary perspective. American Anthropologist 100 (3):632-650.

---. 2013. Biological races in humans. Studies in History and Philosophy of Science Part C: Studies in History and Philosophy of Biological and Biomedical Sciences 44 (3):262-271.

Wade, Nicholas. 2002. Gene study identifies 5 main human populations, linking them to geography. New York Times 20:36.

Winther, Rasmus Grønfeldt, Ryan Giordano, Michael D. Edge, and Rasmus Nielsen. THIS ISSUE. The Mind, the Lab, and the Field: Three Kinds of Populations in Scientific Practice. Studies in History and Philosophy of Biological and Biomedical Sciences TBD:TBD-TBD.

Winther, Rasmus Grønfeldt, and Jonathan Kaplan. 2013. Ontologies and Politics of Biogenomic 'Race'. Theoria 60 (3):54-80. 